\title{
Comparative Evaluation of the Interappointment Pain by Using Different Intracanal Medicaments-An In Vivo Study
}

\author{
Varun Jindal ${ }^{1}$ Sahil Monga ${ }^{1} \quad$ Deepti Jindal ${ }^{1}$ \\ ${ }^{1}$ Department of Conservative Dentistry and Endodontics, Bhojia \\ Dental College and Hospital, Baddi, Himachal Pradesh, India
}

Address for correspondence Varun Jindal, BDS, MDS, \#462, Sector 12, Panchkula, 134109, Haryana, India (e-mail: varundeeptijinda@@gmail.com).

\begin{abstract}
Keywords

- intracanal medicament

- Ledermix paste

- calcium hydroxide

- triple antibiotic paste

Objective The purpose of this in vivo study was to evaluate pain using different intracanal medicaments.

Materials and Methods Seventy-five permanent single-rooted mandibular premolars with acute apical periodontitis were selected for this study. After access cavity preparation and cleaning and shaping of canals, patients were randomly divided into five groups with different intracanal medicaments viz calcium hydroxide paste, calcium hydroxide points, triple antibiotic paste, and Ledermix paste. Interappointment pain was recorded by the patient using visual analog scale after 6 hours, 12 hours, 18 hours, 24 hours, 2 days, 3 days, 4 days, 5 days, and 6 days of intracanal medicament dressing.

Results Ledermix paste showed highly significant results in reducing the interappointment pain as compared with calcium hydroxide paste, calcium hydroxide points, and triple antibiotic paste.

Conclusion Patients with Ledermix as intracanal medicament had minimum interappointment pain.
\end{abstract}

\section{Introduction}

The main objective of endodontic treatment is complete cleaning and shaping of canals followed by threedimensional seal with no discomfort to patients. Sometimes during treatment, patients experience mid-treatment flare-up resulting in pain and/or swelling of oral mucosa or facial soft tissues in the area of tooth being treated. Mid treatment flare-ups can occur because of various reasons viz. overinstrumentation, inadequate debridement of pulp space, periapical extrusion of debris, chemical injuries to periapical tissues due to irrigants, and microbial-induced injury. ${ }^{1}$ Many treatment options have been used to prevent interappointment pain such as drainage through coronal access, proper instrumentation, placing intracanal medicaments, and prescribing analgesics.

The purpose of this in vivo study was to evaluate pain using different intracanal medicaments.

received

January 5, 2019 accepted after revision

January 17, 2019

published online

April 3, 2019
Intracanal medicaments have antibacterial properties. They also enhance the disinfection of prepared root canal by acting as a physical barrier against bacterial penetration. ${ }^{2}$ Many types of intracanal medicaments have been used in endodontics since ages viz. calcium hydroxide, triple antibiotic paste, and Ledermix.

The effectiveness of calcium hydroxide is due to its antimicrobial effect, tissue dissolving property, antiinflammatory action, and osteogenic potential. ${ }^{3}$ Calcium hydroxide can be delivered in the canal in different forms viz. premixed paste, powdered form, and points. Calcium hydroxide points are a relatively recent development and are designed to release calcium hydroxide from a guttapercha matrix.

In an infected root canal, there are many different types of microorganisms, and only one antibiotic cannot act against such a wide range of microflora. Therefore, a combination of antibiotics called triple antibiotic paste has
(C2019 Bhojia Dental College and Hospital affiliated to Himachal Pradesh University

License terms

10.1055/s-0039-1684107

ISSN 2321-1482. 
also been used. It is a combination of three antibiotics: minocycline, ciprofloxacin, and metronidazole. ${ }^{4}$ Though it is effective against Enterococcus faecalis, triple antibiotic paste is shown to be most cytotoxic to human periodontal ligament fibroblasts. It causes exacerbated inflammatory reaction in subcutaneous connective tissue. The minocycline component causes discoloration of the teeth.

Ledermix is a corticosteroid antibiotic paste. Two common active components of Ledermix paste (Riemser) are triamcinolone (a corticosteroid) and demeclocycline (a tetracycline antibiotic). One gram paste contains $30.21 \mathrm{mg}$ demeclocycline calcium (equivalent to $30 \mathrm{mg}$ demeclocycline hydrochloride) and $10 \mathrm{mg}$ triamcinolone acetonide. Triamcinolone is used in the Ledermix materials because of its anti-inflammatory action that assists with rapid pain relief following the commencement of treatment. Demeclocycline is used for its antimicrobial action. ${ }^{5}$

This study was done to evaluate the effectiveness of different intracanal medicament on interappointment pain.

\section{Methodology}

This study was conducted on 75 permanent single-rooted mandibular premolars with acute apical periodontitis in the Department of Conservative Dentistry and Endodontics, Bhojia Dental College, Bhud, Baddi, Himachal Pradesh, from December, 2015 to December, 2017. Approval was granted by ethics committee, and consent form was obtained from each patient prior to treatment.

\section{Inclusion Criteria}

Single-rooted premolars with acute apical periodontitis in the patients of age group 14 to 60 years were selected. The selected teeth were without periapical radiolucency.

\section{Exclusion Criteria}

Teeth with incomplete root formation, severe periodontal problem, and those requiring retreatment were excluded from this study. Patients with systemic problems, for example, diabetes and autoimmune disease, and so on, were not selected for this study.

Before commencing treatment, the complete procedure was explained to the patients and consent was obtained. Local anesthesia was administered, and the tooth was isolated under rubber dam. Access cavity preparation was done, and canals were explored using endodontic explorer (DG-16, Hu Friedy, Chicago, IL). Working length was determined with No. 15 K-files (Mani, Inc., Tochigi, Japan) using apex locator (Root Zx mini apex locator, J. Morita, Kyoto, Japan) and was confirmed with radiovisiography (Kodak Carestream Digital Rvg Sensor 5200, Atlanta, Georgia, United States). Cleaning and shaping were done with K3 files (Sybron endo K3 XF rotary files, California, United States) up to No. 35, 4\% using crown down method along with copious irrigation using 3\% $\mathrm{NaOCl}$ (Prime Dental Products, Bhiwandi, Maharashtra, India) and 0.9\% saline (Alkem Laboratories Pvt. Ltd., Parel, Mumbai, Maharashtra, India). Patients were divided into five groups ( $n=15)$ using randomized sampling technique. Four different medicaments were used for four groups, and one was kept as control. Canals were dried with paper points, and medicaments were placed using lentulospiral into canal according to the groups that are as follows:

Group I (n = 15): Calcium hydroxide paste

Group II ( $\mathbf{n}=\mathbf{1 5})$ : Calcium hydroxide points

Group III ( $\mathbf{n}=\mathbf{1 5})$ : Triple antibiotic paste (mixture of minocycline, ciprofloxacin, and metronidazole in 1:1:1 proportion by $\mathrm{wt}$ )

Group IV ( $\mathbf{n}=\mathbf{1 5})$ : Ledermix paste

Group V ( $\mathbf{n}=\mathbf{1 5})$ : No medicament (control)

Patients were instructed to complete a visual analog scale (VAS) pain score to rate their pain at 6 hours, 12 hours, 18 hours, 24 hours, 2 days, 3 days, 4 days, 5 days, and 6 days after the treatment.

\section{Results}

Change of pain in all groups from 6 hours to 6 days was highly significant (-Fig. 1).

1. From 12 hours to 6 days, all groups had significant difference in change of pain except for group $\mathrm{V}$ that showed significant change in pain at 12 - to 18 -hour interval. However, at 12-hour to 6-day interval, it showed highly significant difference.

2. From 18-hour to 6-day interval, all groups showed highly significant difference except for groups I and III that showed no significant change in pain at interval from 18 to 24 hours.

3. From interval 2 to 6 days, all groups showed highly significant difference in change in pain except

a. for groups I and III that showed no significant difference at interval 2 to 3 days.

b. for group II that showed no significant difference in change in pain at interval 2 to 6 days.

4. At the interval of 3 to 6 days, group 5 showed no significant difference in change in pain.

Change was highly significant in all other four groups from 3- to 6-day interval except

a. for group II that showed no significant difference at 3rd to 5 th days interval

b. for group IV that showed no significant difference at 3 rd to 4 th day interval

5. At the interval of 4 th to 6 th days, change in pain was highly significant in I-IV groups except group $\mathrm{V}$ that showed no significant difference from 4 to 6 days.

6. At the interval of 5 to 6 days, change in pain was not significant in all the groups.

\section{Discussion}

The purpose of using intracanal medicaments is to resolve the infection by maintaining a sterile environment in the prepared root canal system. Thus, intracanal medication may be a valuable adjunct to chemomechanical preparation in the disinfection of the root canal system, reducing the 


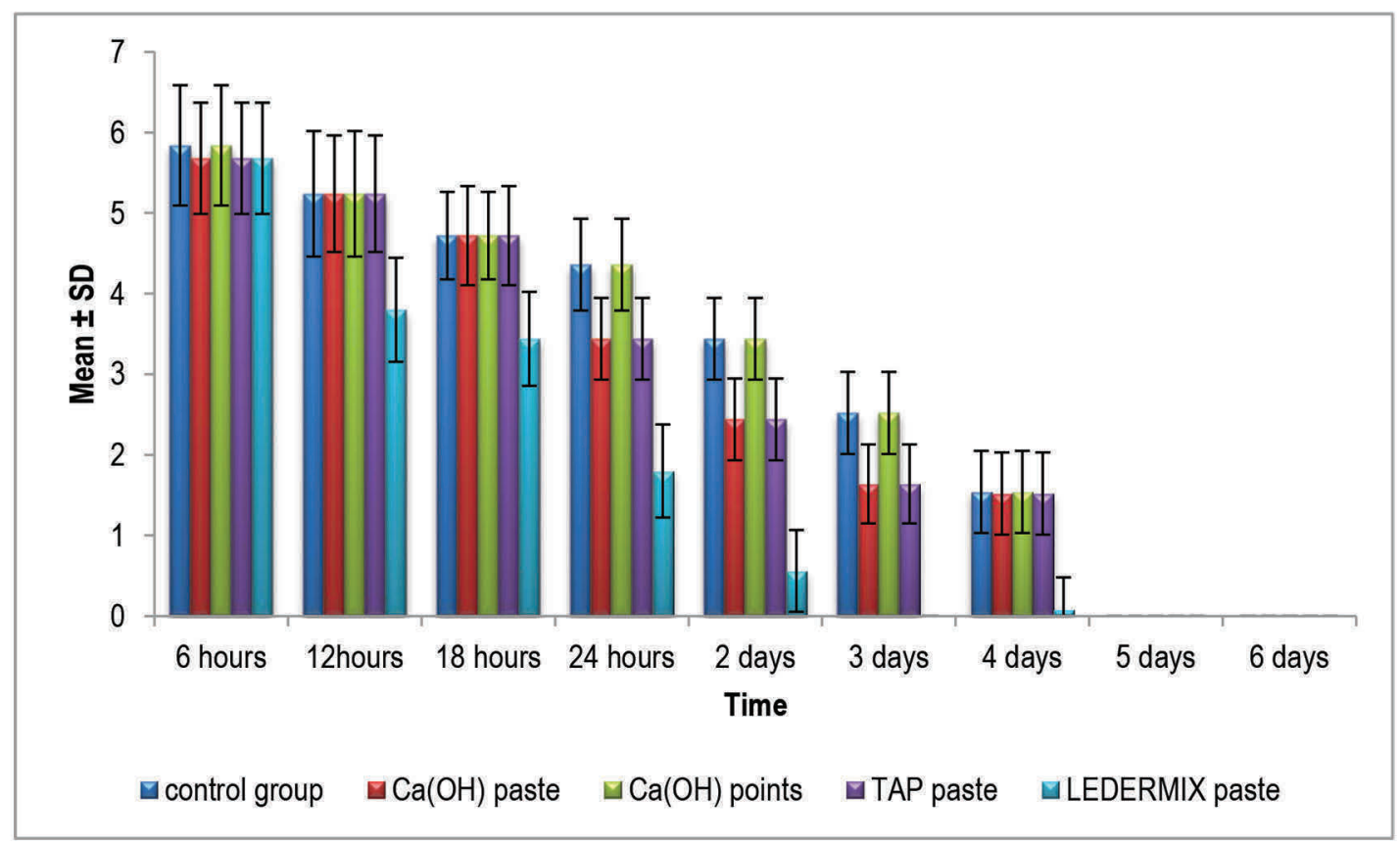

Fig. 1 Comparison of interappointment pain of groups I to V. SD, standard deviation.

endodontic microbiota and therefore favoring periapical tissue repair. ${ }^{6}$

In this study, four different medicaments, that is, calcium hydroxide paste (Apexcal, Ivoclar Vivadent), calcium hydroxide points (Coltene/Waledent, Inc., Germany), triple antibiotic paste, and Ledermix paste (Riemser Pharma GmbH, Germany) were used. These medicaments were compared with each other and then with control group (no medicament) for interappointment pain.

Calcium hydroxide paste is of the most commonly used intracanal medicaments. It has antimicrobial action that functions by ionic dissociation of $\mathrm{Ca}^{2+}$ and $\mathrm{OH}^{-}$ions and induction of hard tissue deposition. The antibacterial action is caused by these ions when they act upon vital tissue and bacteria. ${ }^{7-9}$ In calcium hydroxide points, pure calcium hydroxide is homogeneously distributed throughout a gutta-percha matrix. Solubility of calcium hydroxide and the mobility of the ions get improved by sodium chloride and surfactant. Small amount of calcium hydroxide is continuously released from these points..$^{10}$ Elevated $\mathrm{pH}$ of above 12 is maintained by continuous release of calcium hydroxide ions that act on cell membrane and DNA of microorganisms causing cell death.

Triple-antibiotic paste contains ciprofloxacin, metronidazole, and minocycline. Metronidazole is a nitroimi-dazole compound that exhibits broad spectrum of activity against protozoa and anaerobic bacteria. It is selectively toxic to anaerobic microorganisms. Minocycline is primarily bacteriostatic that inhibits protein synthesis. It exhibits broad-spectrum activity against gram-positive and gram-negative microorganisms. Ciprofloxacin is a synthetic fluoroquinolone with rapid bactericidal action. ${ }^{11}$ The concern of the triple antibiotic paste is that it may cause bacterial resistance. Additionally, minocycline may cause tooth discoloration. ${ }^{12}$

To overcome this disadvantage of triple-antibiotic paste, Ledermix paste was introduced. Its main composition is triamcinolone (a corticosteroid) and demeclocycline (a tetracycline antibiotic). Triamcinolone has an antiinflammatoryactionthatgivespatientrelieffromposttreatment pain. Demeclocycline is used for its antimicrobial action. Ledermix paste is a good option to be placed in root canal system to manage acute irreversible pulpitis after removing the inflamed pulp. ${ }^{13}$ Triamcinolone that has anti-inflammatory action acts on inflamed peri-radicular area that may have resulted due to overinstrumentation. Therefore, its direct action on these tissues decreases the inflammation. Furthermore, the demeclocycline has antimicrobial effect that will act against microflora. ${ }^{14,15}$

This study showed significant results in favor of Ledermix paste similar to the study done by Ehrmann et al. ${ }^{16}$ They investigated the relationship of postoperative pain to three different medicaments placed in the root canal after a complete biomechanical debridement of the root canal system in patients presenting for emergency relief of pain. They concluded that patients with painful teeth with acute apical periodontitis that were given dressing of Ledermix paste experienced less pain than those with calcium hydroxide, triple-antibiotic paste, or no dressing.

This study results correspond to the study results conducted by Dall et $\mathrm{al}^{17}$ which states that there was a significant difference in the interappointment pain at 48-hour interval in the group with Ledermix paste as intracanal medicament when compared with calcium hydroxide.

Trope $^{18}$ showed no significant difference between different intracanal medicaments viz formocresol, 
calcium hydroxide, and Ledermix paste. It could be due to difference in the methodology of canal preparation or difference in the concentration of irrigants used. Moreover, in his study ibuprofen was used for postoperative pain control that could cause change of pain perception of different patients.

In this study, there was no significant difference in decreasing rate of pain among all the groups except Ledermix paste. Ledermix started showing decrease in pain after 6 hours only, and most patients of Ledermix group showed no pain at second and third days.

\section{Conclusion}

This study results concluded that Ledermix showed better results compared to other intracanal medicaments during multi-visit root canal treatment in decreasing the interappointment pain. This study outcome holds up the potentialvalue of theintracanal use of corticosteroid-antibiotic combination for the relief of interappointment pain, and further studies should be done to check its advantages over other medicaments in different conditions.

\section{Limitations}

In this study, patients having only acute apical periodontitis were included. Further studies should also be done to compare these medicaments in other conditions such as periradicular lesions, etc.

\section{Conflict of Interest}

None declared.

\section{References}

1 Shetty N. Mid treatment flareups in endodontics-a dilemma. 2005; xx:20-23

2 Bansal R, Jain A. Overview on the current antibiotic containing agents used in endodontics. $\mathrm{N}$ Am J Med Sci 2014;6(8):351-358

3 Fava LR, Saunders WP. Calcium hydroxide pastes: classification and clinical indications. Int Endod J 1999;32(4):257-282
4 Vijayaraghavan R, Mathian VM, Sundaram AM, Karunakaran R, Vinodh S. Triple antibiotic paste in root canal therapy. J Pharm Bioallied Sci 2012;4(2):230-233

5 Ahmed HM, Abbott PV. Discolouration potential of endodontic procedures and materials: a review. Int Endod J 2012;45(10):883-897

6 Abbott PV. Medicaments: aids to success in endodontics. Part 1. A review of the literature. Aust Dent J 1990;35(5):438-448

7 Mohammadi Z, Dummer PM. Properties and applications of calcium hydroxide in endodontics and dental traumatology. Int Endod J 2011;44(8):697-730

8 Lohbauer U, Gambarini G, Ebert J, Dasch W, Petschelt A. Calcium release and $\mathrm{pH}$-characteristics of calcium hydroxide plus points. Int Endod J 2005;38(10):683-689

9 Estrela C, Rodrigues de Araújo Estrela C, Bammann LL, Pecora JD. Two methods to evaluate the antimicrobial action of calcium hydroxide paste. J Endod 2001;27(12):720-723

10 Siqueira JF Jr, de Uzeda M. Intracanal medicaments: evaluation of the antibacterial effects of chlorhexidine, metronidazole, and calcium hydroxide associated with three vehicles. J Endod 1997;23(3):167-169

11 Varalakshmi R, Parasuraman BSM. 3Mix-MP in endodontics-an overview. IOSR-JDMS 2012;3(1):36-45

12 Kim JH, Kim Y, Shin S-J, Park J-W, Jung IY. Tooth discoloration of immature permanent incisor associated with triple antibiotic therapy: a case report. J Endod 2010;36(6):1086-1091

13 Chu FC, Leung WK, Tsang PC, Chow TW, Samaranayake LP. Identification of cultivable microorganisms from root canals with apical periodontitis following two-visit endodontic treatment with antibiotics/steroid or calcium hydroxide dressings. J Endod 2006;32(1):17-23

14 Negm MM. Intracanal use of a corticosteroid-antibiotic compound for the management of posttreatment endodontic pain. Oral Surg Oral Med Oral Pathol Oral Radiol Endod 2001;92(4):435-439

15 Iranmanesh F, Parirokh M, Haghdoost AA, Abbott PV. Effect of corticosteroids on pain relief following root canal treatment: a systematic review. Iran Endod J 2017;12(2):123-130

16 Ehrmann EH, Messer HH, Adams GG. The relationship of intracanal medicaments to postoperative pain in endodontics. Int Endod J 2003;36(12):868-875

17 Dall AQ, Jouhar R, Khoso NA. Comparison of inter-appointment pain between Ledermix and no intracanal medicament in acute apical periodontitis. J Liaquat Uni Med Health Sci 2011;10(3):106-111

18 Trope M. Relationship of intracanal medicaments to endodontic flare-ups. Endod Dent Traumatol 1990;6(5):226-229 